\section{Changing patent laws could be a healthy move to combat resistance}

Sir-A contradiction exists with the commercial development of chemotherapeutics such as antibiotics, anthelmintics and antiprotozoal agents, and the generation of resistance in organisms to these agents, for which I would like to suggest a solution.

Chemotherapeutic companies invest large amounts of money and effort in research, registration and marketing of new products. This investment is usually protected for 15 years after patent acceptance. However, by the time the product reaches the market the patent period has partially expired (even though in many cases companies are able to recoup investment through additional patent claims and similar patenting strategies).

In some cases the company needs to have optimized its return on investment by the time the patent expires and generic products appear on the market. This means that there is considerable commercial pressure on the company to achieve the greatest possible market penetration and sales volume to service the investment before this time.

As well, in the increasingly global freemarket economy that we live in,

chemotherapeutic companies are under an obligation to maximize profits for their shareholders, both in the short term and in the long term.

Chemotherapeutic companies have to promote their product in the most effective and efficient manner to ensure their own commercial survival and to maximize the return on their investment, while at the same time trying to husband the use of their products for the greater long-term public good.

This task is a paradoxical challenge for manufacturers, national registration agencies and governments. The use of these agents, as has been repeatedly demonstrated, results in the selection of existing resistant organisms within the population concerned, and/or creates favourable conditions for resistant mutants to thrive.

The cycle of short-term commercial pressure for optimal market return on investment and the resultant generation of resistance should be broken, if possible, in the long-term interests of human and animal health.

A radical and self-regulatory approach to break this cycle could be to link the continuation of a patent to the resistance status of the product. This approach would encourage marketing strategies and patterns of use that are designed to preserve the effectiveness of the preparation and to minimize the development of resistance.

There are likely to be many and varied practical, theoretical and ideological objections to this proposal. Examples are that it is too difficult to change the patent laws and that the nature of the development of resistance is unpredictable.

Is it possible to address this paradox without a lateral approach, such as altering patent laws internationally and reducing the commercial pressure for short-term commercial return on investment?

Any new agents for the chemotherapeutic treatment of bacterial, parasitic and protozoal diseases need to be conserved, if possible, for future generations. To date, the evidence for longevity of the effectiveness of many existing drugs in medicine and animal health has not been good.

Terry Nicholls

11 Poole Place, Latham ACT 2615, Australia

\section{We need both computer models and experiments}

Sir - We were delighted by your News report "Computer modellers seek out 'Ten Most Wanted' proteins" (Nature 409, 4; 2001), highlighting recent advances in protein structure prediction.

The report states, however, that computational structural biologists "believe that computer modelling may become a viable alternative to the current practice of using X-ray crystallography and nuclear magnetic resonance techniques to 'solve' the structure of a protein".

The importance and value of structure prediction methods is to produce rough, approximate models for proteins with no experimental structure available. These models can often provide verifiable hypotheses about function, as your report accurately mentions. However, in the forseeable future we expect predicted structures neither to approach the accuracy of experimentally determined structures, nor to replace them.

The report also states: "The big question, researchers say, is whether modelling will continue to improve fast enough to make an impact. There is a relatively short window of time for structure prediction to be useful before structural genomics ... generates all the results needed."

We disagree strongly with this statement. Structural genomics aims not to solve the structures of all proteins, but rather to obtain a set of representative structures such that all others can be modelled. Thus computational methods will play a critical role in translating the information on the relatively small fraction of proteins whose structures will be solved into accurate models for all proteins.

We furthermore expect that computational methods will produce useful low-resolution models for large numbers of sequence families many years before representative structures can be solved experimentally, and will play an all-important role in the design of novel proteins and therapeutics.

Daniel Fischer*, David Baker $†$, John Moult ${ }^{*}$ Bioinformatics, Department of Computer Science, Ben Gurion University, Beer-Sheva 84015, Israel $\dagger$ Department of Biochemistry and Howard Hughes Medical Institute, Box 357350, University of Washington, Seattle, Washington 98195, USA \$Centre for Advanced Research in Biotechnology, University of Maryland Biotechnology Institute, Rockville, Maryland 20850, USA

\section{Why Pauling didn't solve the structure of DNA}

Sir - Linus Pauling, who was celebrated in Gautam R. Desiraju's Millennium Essay (Nature 408, 407; 2000), was born on 28 February 1901. As the hundredth anniversary of his birth approaches, I would like to describe Pauling's comments on the discovery of the structure of DNA.

Shortly before Christmas 1988, Linus was the keynote speaker at the UCLA-Sloan winter school on molecular evolution. He struck up an immediate rapport with my wife, Laura, showing her pictures of his late wife and of his son as a toddler. As we were leaving his lecture to take him back to the faculty guest house, he suddenly asked my wife and me in his uniquely direct way if we ever wondered "why he hadn't solved the structure of DNA".

We were totally surprised by this, but at the same time were curious to know the answer and asked him why. He said that one day his wife had asked him that question. It had made him think and he replied something to the effect of "I don't know, I guess that I always thought that the DNA structure was mine to solve, and therefore I didn't pursue it aggressively enough".

Perhaps this isn't the only reason, but on that December night it was Linus's reason. His presence is missed. Jim Lake

Molecular Biology Institute and MCD Biology, UCLA, Los Angeles, California 90095, USA 